Pacific Journal of Mathematics

ON A CONJECTURE OF H. HADWIGER 


\title{
ON A CONJECTURE OF H. HADWIGER
}

\author{
BRANKO GRÜNBAUM
}

1. For any convex body (i.e., compact convex set with interior points) $K$ in the Euclidean plane $E^{2}$ let $i(K)$ denote the greatest integer with the following property:

There exist translates $K_{n}, 1 \leqq n \leqq i(K)$, of $K$ such that

$$
\begin{array}{ll}
K \cap K_{n} \neq \phi & \text { for all } n ; \\
\text { Int } K_{n} \cap \text { Int } K_{m}=\phi & \text { for } n \neq m .
\end{array}
$$

It is well known (see e.g., Hadwiger [3]) that $7 \leqq i(K) \leqq 9$ for any $K \subset E^{2}{ }^{1}$ and that the bounds are attained (e.g., $i(K)=7$ if $K$ is a circle, $i(K)=9$ if $K$ is a parallelogram). Hadwiger conjectured, ${ }^{2}$ moreover, that if $K$ is not a parallelogram, then $i(K)=7$.

We shall establish Hadwiger's conjecture in the following theorem:

If $K$ is not a parallelogram, then $i(K)=7$. Moreover, if 7 translates o $K$ satisfy conditions (1) then one of them coincides with $K$.

In the proof we shall use some results on centrally symmetric convex sets; they are collected in $\S 2$. The proof of the theorem follows in $\S 3$. In $\S 4$ we make some remarks on related problems in higherdimensional spaces. $\$ 5$ contains some results on the related problem on the number of translates of a convex set needed to "enclose" the set.

2. Let $K$ be any centrally symmetric plane convex body with the origin 0 as center. Then a Minkowski geometry, with norm \|\| , is defined in the plane, for which $K$ is the unit cell.

We note the following propositions:

(i) For any point $x$ with $\|x\|=1$ there exist points $y, z$ satisfying $\|y\|=\|z\|=\|x-y\|=\|y-z\|=\|x+z\|=1$. (In other words, any $x \in$ Front $K$ is a vertex of at least one affine-regular hexagon whose vertices belong to Front $K$ ).

(ii) Let $x, y, z$ be different points belonging to Front $K$, such that the origin 0 does not belong to that open half-plane determined by $x$ and $y$ which contains $z$. Then $\|x-y\| \geqq\|x-z\|$, with equality taking place only in case $y, z$, and $(y-x) /\|y-x\|$ belong to a straight-line segment contained in Front $K$.

Received November 3, 1958. This research was supported by the United States Air Force through the Air Force Office of Scientific Research of the Air Research and Development Command, under contract No. AF 49(637)-253. Reproduction in whole or in part is permitted for any purpose of the United States Government.

1 Related results, pertaining to more general sets, are given in [4].

2 Oral communication from Dr. H. Debrunner. 
(iii) Let $x, y, z, u$ be different points belonging to Front $K$, such that $z$ and $u$ belong to an open half-plane determined by $x$ and $y$, while 0 belongs to its complement. Then either $\|x-y\|=\|z-u\|=2$ or $\|x-y\|>\|z-u\|$.

Proofs of (i) have been given in [5], [6], [9]; (ii) and (iii) are proved in [2].

(iv) Let $y_{i}, 1 \leqq i \leqq 8$, be such that $\left\|y_{i}\right\|=1,\left\|y_{i}-y_{j}\right\| \geqq 1$ for $i \neq j$. Then $K$ is a parallelogram.

Proof. Since in Minkowski geometry a straight-line segment is a path of minimal length between two points, the above hypotheses imply that the perimeter of $K$ is $\geqq 8$ (in the Minkowski metric). But it is well known (see, e.g., [6], [9]) that the unit cell of any Minkowski plane has a perimeter $\leqq 8$; moreover, the same proofs easily yield also the fact that the perimeter equals 8 only if $K$ is a parallelogram, which ends the proof of (iv).

(v) If there exists a set $Y=\left\{y_{i}, 1 \leqq i \leqq 7\right\} \subset$ Front $K$ such that $\left\|y_{i}-y_{j}\right\| \geqq 1$ for $i \neq j$, then $K$ is a parallelogram.

Proof. Let $\pm x_{i}, i-1,2,3$, be the vertices of any affine-regular hexagon $H$ inscribed in $K$ (such hexagons exist by (i)). We note that:

(a) If two points of $Y$ are opposite vertices of $H$, then $Y \cup(-Y)$ contains 8 points satisfying the assumptions of (iv), and therefore $K$ is a parallelogram;

(b) No pair of points $y_{i}, y_{j} \in Y$ can belong to the interior of a small arc of Front $K$ determined by two neighboring vertices of $H$, since in such a case (iii) would imply that $\left\|y_{i}-y_{j}\right\|<1$.

Now, if (a) does not hold, it is clear that we may find $H$ such that, after suitably changing the indices if necessary, the following relations hold ( $<$ denotes equality, or precedence according to a fixed orientation of Front $K)$ :

$$
x_{1}=y_{1} \prec y_{2} \prec x_{2} \prec y_{3} \prec x_{3} \prec y_{4} \prec-x_{1}, y_{2} \neq x_{2}, y_{4} \neq-x_{1} .
$$

Then (ii) implies that $\left\|y_{1}-y_{2}\right\|=1$, and that $y_{2}, x_{2}, y_{3}, x_{3}$ belong to a maximal straight-line segment $[a, b] \subset$ Front $K$, with $x_{1} \prec a \prec x_{2}$. Now, if $y_{4} \in[a, b]$ we have $\|a-b\| \geqq 2$ which establishes $K$ as a parallelogram. Let us therefore assume $y_{4} \notin[a, b]$. Jointly with $y_{4} \neq-x_{1}$ this implies that $y_{2}=a, y_{3}=a-x_{1}$, and $\left\|y_{3}-y_{2}\right\|=1$, since otherwise the affine-regular hexagon with vertices $\pm x_{1}, \pm a, \pm\left(a-x_{1}\right)$ would yield the situation described in (b). Now $\left\|y_{3}-\left(-x_{1}\right)\right\|=\|a\|=1$ and $\left\|y_{3}-y_{4}\right\| \geqq 1$ imply, by (ii), that $\left\|y_{3}-y_{4}\right\|=1$ and that $y_{4},-x_{1}$, and $-a$ are points of a segment $[-a, c] \subset$ Front $K$, which is obviously adjacent to the segment $[-a,-b]$. 
Using (ii) repeatedly we see that $-x_{1} \prec y_{5}$ and $y_{5} \neq-x_{1}$; therefore $-b-x_{1} \prec y_{6}$ and $y_{6} \neq-b-x_{1}$, so $-b \prec y_{7}$ with $y_{7} \neq-b$. But this is impossible since it would imply $\left\|y_{1}-y_{7}\right\|<\left\|x_{1}+b\right\|=1$. Accordingly, $y_{4}$ must belong to $[a, b]$, and (v) is proved.

(vi) If $P=-P$ is a parallelogram, if $C$ is a convex set, and if $P=(1 / 2)[C+(-C)]$, then $C=P+x$ for a suitable point $x$.

Proof. Considering the supporting lines of $P$ it is immediate that $C$ must be a parallelogram with sides parallel to those of $P$; therefore $P=(1 / 2)[C+(-C)]$ implies that $C$ is a translate of $P$.

Remark. The author is indebted to Professor E. G. Straus for the remark that (vi) has to be used in order to complete the original proof of the theorem. Professor Straus also observed that if $K$ is a centrally symmetric plane convex body different from a parallelogram, then $K=(1 / 2)[C+(-C)]$ for some $C$ which is not a translate of $K$. The following particularly simple proof of this fact was given by Dr. E. Asplund:

Inscribe an affine regular hexagon $H$ in $P$ (see (i)) and construct a curve $(1 / 2) C$ consisting of translates of the arcs of the boundary of $P$ which are determined by alternate sides of $H$. It is easy to see that $(1 / 2) C$ is not homothetic to the boundary of $P$ unless $P$ is a parallelogram. On the other hand $(1 / 2) C$ has constant width 1 in the Minkowski metric whose unit sphere is $P$ (it is in fact a Reuleux triangle for that metric) and thus $-(1 / 2) C+(1 / 2) C$ is the sphere $P$ as the only centrally symmetric body of constant width.

The related question of non-trivial decomposability of centrally symmetric convex bodies in three-dimensional space seems to be much more complicated. Using results of Gale [1] it is easily established that parallelepipeds, octahedra and other centrally-symmetric anti-prisms, as well as other sets, are only trivially decomposable in the form (1/2)[C+ $(-C)]$.

3. We now turn to the proof of our theorem. First of all we remark that without loss of generality we may assume $K$ to be centrally symmetric. Indeed, if $K$ is any convex set, $(1 / 2)[K+(-K)]$ is centrally symmetric; but, as has been noted by Minkowski [8] and used also by Hadwiger [3], $(x+K) \cap(y+K)$ and $(x+(1 / 2)[K+(-K)]) \cap$ $(y+(1 / 2)[K+(-K)])$ are simultaneously empty, non-empty, or have interior points. Therefore, (vi) implies that the general case follows from the symmetric one.

Assuming now that $K$ is centrally symmetric and that the translates $K_{n}=z_{n}+K$ satisfy conditions (1), we construct a new family of translates $\left\{K_{n}^{*}\right\}$ as follows: If $z_{n}=0$ we put $K_{n}^{*}=K_{n}$; if $z_{n} \neq 0$, we define $K_{n}^{*}=\left(2 z_{n} /\left\|z_{n}\right\|\right)+K$. The family $K_{n}^{*}$ then satisfies the conditions 
(1). Indeed, $K_{n}^{*} \cap K$ obviously contains $y_{n}=z_{n} /\left\|z_{n}\right\|$ (resp. $y_{n}=0$ if $z_{n}=0$ ), and for $n \neq m$ we have

$$
\text { Int } K_{n}^{*} \cap \operatorname{Int} K_{m}^{*}=\phi
$$

since (1), assumed to hold for the family $\left\{K_{n}\right\}$, implies

$$
\operatorname{Int}\left(\lambda z_{n}+K\right) \cap \operatorname{Int}\left(\mu z_{m}+K\right)=\phi \text { for any } \lambda, \mu \geqq 1 \text {. }
$$

Now, (2) implies that $\left\|2 y_{n}-2 y_{m}\right\| \geqq 2$, i.e., $\left\|y_{n}-y_{m}\right\| \geqq 1$, and therefore the theorem follows from (v).

4. The number $i(K)$ may be defined in the same way for convex bodies in any Euclidean space. Hadwiger proved that $i(K) \leqq 3^{k}$ for $K \subset E^{k}$, the bound being attained for $k$-dimensional parallelotopes. On the other hand we have:

If $K \subset E^{k}$ then $i(K) \geqq k^{2}+k+1$.

Proof. As above, we may without loss of generality assume that $K$ is centrally symmetric with center 0 . Let the points $x_{i}, 0 \leqq i \leqq k$, satisfy $\left\|x_{i}-x_{j}\right\|=2$ for $i \neq j$, where the norm is taken in the Minkowski metric determined by $K$. (The existence of such a family $\left\{x_{i}\right\}$ may be established by obvious continuity arguments.) Then the $k^{2}+k+1$ sets $x_{i}-x_{j}+K$, for $0 \leqq i, j \leqq k$, satisfy conditions (1). Thus our assertion is established.

The above estimate $i(K) \geqq k^{2}+k+1$ is the best possible; it is attained if $K$ is, e.g., a simplex. This is obvious for $k \leqq 3$, and may be established also in the general case.

As a generalization of the result of $\S 1$, we conjecture that $i(K)$ is odd for any $K$ and that any odd value between $k^{2}+k+1$ and $3^{k}$ is assumed. The last part of the conjecture is easily verified for $k=3$.

5. We end with a related result. Following [4], we shall say that a set $A$ encloses a set $B$ if every unbounded connected set which intersects $B$ also intersects $A$. For any convex body $K$ in the Euclidean plane let $e(K)$ denote the smallest natural number with the property:

There exist translates $K_{n}, 1 \leqq n \leqq e(K)$, such that

$$
\begin{aligned}
& \text { Int } K \cap \text { Int } K_{n}=\phi \text { for all } n \\
& \bigcup_{n=1}^{e(K)} K_{n} \text { encloses } K .
\end{aligned}
$$

With this terminology we have

If $K$ is not a parallelogram, then $e(K)=6$. For a parallelogram $P, e(P)=4$.

This result may be established by the same methods we used in 
$\S \S 2$ and 3 . Using the conventions of $\S 2$, the main step of the proof (which is used instead of (iv) and (v)) may be formulated as follows:

(vii) If $Y=\left\{y_{i} ; 1 \leqq i \leqq 5\right\} \subset$ Front $K$ with $\left\|y_{i}-y_{i+1}\right\| \leqq 1$ and $y_{i} \prec y_{i+1}$ for all $i\left(y_{6}=y_{1}\right)$, and if the origin belongs to the convex hull of $Y$, then $K$ is a parallelogram.

We may also mention another theorem of a similar kind, established by Levi [7]: If $K$ is a convex body in the plane, different from a parallelogram, then there exist three translates of Int $K$ such that their union covers $K$ (and therefore encloses it). For centrally symmetric sets a stronger theorem of the same type is given in [2].

\section{REFERENCES}

1. D. Gale, Irreducible convex sets, Proc. Congr. Amsterdam, 2, (1954), 217-218.

2. B. Grünbaum, Borsuk's partition conjecture in Minkowski planes, Bull. Research Council of Israel, 7F (1957), 25-30.

3. H. Hadwiger, Ueber Treffanzahlen bei translationsgeichen Eikörpern, Archiv der Mathematik, 8 (1957), 212-213.

4. C. J. A. Halberg, Jr., E. Levin, and E. G. Straus, On contiguous congruent sets in Euclidean space, Proc. Amer. Math. Soc., 10 (1959), 335-344.

5. P. J. Kelly, A property of Minkowskian circles, Amer. Math. Monthly, 57 (1950), 677678.

6. D. Laugwitz, Konvexe Mittelpunktsbereiche und normierte Räume, Math. Zeitschr., 61 (1954), 235-244.

7. F. W. Levi, Ueberdeckung eines Eibereiches durch Parallelverschiebungen seines offenen Kerns, Archiv der Math., 6 (1955), 369-370.

8. H. Minkowski, Dichteste gitterformige Lagerung kongruenter Körper, Nachr. der K. Ges. der Wiss. zu Göttingen, Math.-Phys. Kl., 1904, 311-355 (=Gesam. Abh., Vol. 2, Leipzig und Berlin, 1911, pp. 3-42).

9. Yu. G. Rešetnyak, An extremal problem from the theory of convex curves, Uspehi Matem. Nauk (N. S.) 8 No. 6 (58) (1953), 125-126. (Russian)

The Institute For Advanced Study

PRINCETON, NEW JERSEY 



\section{PACIFIC JOURNAL OF MATHEMATICS}

\section{EDITORS}

Ralph S. Phillips

Stanford University

Stanford, California

F. H. BrownelL

University of Washington

Seattle 5 , Washington
A. L. Whiteman

University of Southern California Los Angeles 7. California

L. J. PAIGE

University of California

Los Angeles 24, California

ASSOCIATE EDITORS
E. F. BECKENBACH
D. DERRY
H. L. ROYDEN
E. G. STRAUS
T. M. CHERRY
M. OHTSUKA
E. SPANIER
F. WOLF

\section{SUPPORTING INSTITUTIONS}

UNIVERSITY OF BRITISH COLUMBIA

CALIFORNIA INSTITUTE OF TECHNOLOGY

UNIVERSITY OF CALIFORNIA

MONTANA STATE UNIVERSITY

UNIVERSITY OF NEVADA

NEW MEXICO STATE UNIVERSITY

OREGON STATE COLLEGE

UNIVERSITY OF OREGON

OSAKA UNIVERSITY

UNIVERSITY OF SOUTHERN CALIFORNIA
STANFORD UNIVERSITY

UNIVERSITY OF TOKYO

UNIVERSITY OF UTAH

WASHINGTON STATE COLLEGE,

UNIVERSITY OF WASHINGTON

AMERICAN MATHEMATICAL SOCIETY

CALIFORNIA RESEARCH CORPORATION

HUGHES AIRCRAFT COMPANY

SPACE TECHNOLOGY LABORATORIES

NAVAL ORDNANCE TEST STATION

Printed in Japan by International Academic Printing Co., Ltd., Tokyo, Japan

Reprinted 1966 in the United States of America 


\section{Pacific Journal of Mathematics}

\section{Vol. 11, No. $1 \quad$ November, 1961}

A. A. Albert, Generalized twisted fields ............................ 1

Richard Arens, Operational calculus of linear relations ................... 9

John Herbert Barrett, Disconjugacy of a self-adjoint differential equation of the fourth order ....................................... 25

Paul Richard Beesack, Hardy's inequality and its extensions ............... 39

Julius Rubin Blum and David Lee Hanson, On invariant probability measures.

II .............................................

Robert Allen Bonic, Symmetry in group algebras of discrete groups.......... 73

R. Creighton Buck, Multiplication operators ...................... 95

Jack Gary Ceder, Some generalizations of metric spaces ................. 105

Meyer Dwass, Random crossings of cumulative distribution functions ......... 127

Albert Edrei, Wolfgang H. J. Fuchs and Simon Hellerstein, Radial distribution and

deficiencies of the values of a meromorphic function ............... 135

William Cassidy Fox, Harmonic functions with arbitrary local singularities ..... 153

Theodore Thomas Frankel, Manifolds with positive curvature ............... 165

Avner Friedman, A strong maximum principle for weakly subparabolic

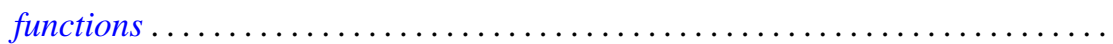

Watson Bryan Fulks and J. O. Sather, Asymptotics. II. Laplace's method for

multiple integrals ......................................

Adriano Mario Garsia and Eugene Richard Rodemich, An embedding of Riemann

surfaces of genus one ..................................... 193

Irving Leonard Glicksberg, Weak compactness and separate continuity......... 205

Branko Grünbaum, On a conjecture of H. Hadwiger .................. 215

Frank J. Hahn, On the action of a locally compact group on $E_{n} \ldots \ldots \ldots \ldots \ldots . . \ldots 221$

Magnus R. Hestenes, Relative hermitian matrices ..................... 225

G. K. Kalisch, On similarity invariants of certain operators in $L_{p} \ldots \ldots \ldots \ldots .247$

Yitzhak Katznelson and Walter Rudin, The Stone-Weierstrass property in Banach

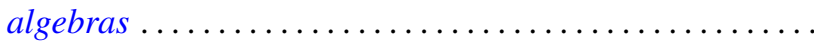

Samir A. Khabbaz, The subgroups of a divisible group $G$ which can be represented as intersections of divisible subgroups of $G \ldots \ldots \ldots \ldots \ldots \ldots \ldots \ldots \ldots . \ldots \ldots 7$

Marvin Isadore Knopp, Construction of a class of modular functions and forms .......................................... 275

Charles Alan McCarthy, Commuting Boolean algebras of projections .......... 295

T. M. MacRobert, Transformations of series of E-functions ................ 309

Heinz Renggli, An inequality for logarithmic capacities ................. 313

M. S. Robertson, Applications of the subordination principle to univalent functions .......................................... 315

David Sachs, Partition and modulated lattices ..................... 325

Frank S. Scalora, Abstract martingale convergence theorems ............... 347

Elbert A. Walker, Torsion endomorphic images of mixed Abelian groups ........ 375

Morgan Ward, The prime divisors of Fibonacci numbers................. 379

Charles R. B. Wright, On the nilpotency class of a group of exponent four....... 387 